Helical ITER hybrid scenario equilibria

This article has been downloaded from IOPscience. Please scroll down to see the full text article.

2011 Plasma Phys. Control. Fusion 53024002

(http://iopscience.iop.org/0741-3335/53/2/024002)

View the table of contents for this issue, or go to the journal homepage for more

Download details:

IP Address: 128.178.125.164

The article was downloaded on 02/02/2011 at $14: 11$

Please note that terms and conditions apply. 


\title{
Helical ITER hybrid scenario equilibria
}

\author{
W A Cooper, J P Graves and O Sauter
}

Ecole Polytechnique Fédérale de Lausanne (EPFL), Centre de Recherches en Physique des Plasmas, Association Euratom-Confédération Suisse, CH1015 Lausanne, Switzerland

Received 29 June 2010, in final form 10 September 2010

Published 20 January 2011

Online at stacks.iop.org/PPCF/53/024002

E-mail: wilfred.cooper@epfl.ch

\begin{abstract}
Numerical computations of ITER equilibria in the hybrid scenario using a three-dimensional (3D) magnetohydrodynamic equilibrium code with nested magnetic flux surfaces demonstrate the formation of internal 3D helical cores similar to saturated ideal internal kink modes under reversed magnetic shear conditions when the minimum value of the inverse rotational transform is in the neighbourhood of unity.
\end{abstract}

(Some figures in this article are in colour only in the electronic version)

\section{Introduction}

The 'snakes' in JET [1], the continuous mode in MAST [2] and the single helical axis (SHax) configurations in the RFX [3] reversed field pinch constitute manifestations that nominally axisymmetric systems can spontaneously develop three-dimensional (3D) internal equilibrium structures that are not captured with standard tokamak equilibrium solvers. Typically until now, nonlinearly saturated mode structures have been calculated analytically [4] and with nonlinear magnetohydrodynamic (MHD) codes [5]. Alternatively, introducing a small forcing term in the second variation of the energy principle, then removing it after many iterations, can lead to bifurcated solutions with 3D characteristics [6]. This suggests that the application of 3D equilibrium codes developed for stellarator applications should be considered to model this type of phenomena in tokamaks. The SHax state in RFX has already been successfully modelled with the VMEC code [7] in which an internal helical structure with seven-fold periodicity corresponding to the lowest order core resonant surface has been computed [8]. Similarly, internal helical structures have been calculated with the ANIMEC code [9] in elongated high current TCV-like tokamak configurations with reversed or very flat inverse rotational transform $q$-profiles with $q_{\min } \sim 1$ [10].

Nonlinear tokamak MHD codes (like that of [5]) develop four possible solution states:

- The plasma is stable linearly (and nonlinearly) and the perturbations remain infinitesimally small. 
- The plasma is violently unstable, the displacement vector becomes very large with a strong stochastization of the magnetic field lines. This would model a disruptive termination of an experimental tokamak discharge.

- The plasma evolves into a saturated state with distinct island structures separated by closed magnetic flux surfaces.

- The plasma, after an initial unstable linear stage, saturates in a nonlinear 3D quasi-static state in which the perturbed structure does not extend to the edge of the plasma. We argue that the equilibrium model we apply is capable of essentially reproducing static configurations of this type.

In this paper, we explore the possibilities of an ITER hybrid scenario configuration $[11,12]$ to develop core helical deformations.

\section{The MHD equilibrium state}

The minimization of the energy $W$,

$$
\mu_{0} W=\iiint \mathrm{d}^{3} x\left(\frac{B^{2}}{2}+\frac{\mu_{0} p_{\|}}{\Gamma-1}\right),
$$

constitutes the starting point for the computation of 3D MHD equilibrium states. The magnetic field strength is $B$, the parallel total plasma pressure is $p_{\|}, \mu_{0}$ is the permeability of free space and $\Gamma$ is the adiabatic index. The total parallel pressure can be expressed in the form [9]

$$
p_{\|}(s, B)=\mathcal{M}(s)\left[\Phi^{\prime}(s)\right]^{\Gamma} \frac{1+p_{\mathrm{h}}(s) H(s, B)}{\left\langle 1+p_{\mathrm{h}}(s) H(s, B)\right\rangle^{\Gamma}},
$$

where $\mathcal{M}$ is the plasma mass, $p_{\mathrm{h}}$ is a hot particle pressure amplitude factor and $H(s, B)$ is a form factor that governs the variation of the hot particle pressure components around each flux surface labelled with radial variable $s, 0 \leqslant s \leqslant 1$. The coordinate $s$ is proportional to the toroidal magnetic flux function $\Phi$ (normalized to its value at the plasma-vacuum interface). The symbol prime $\left({ }^{\prime}\right)$ indicates the derivative of a flux surface quantity with respect to $s$ and $\langle\ldots\rangle$ denotes a flux surface average. Invoking a bi-Maxwellian model to describe the hot particle distribution function [13], the form function $H(s, B)$ reads as [9, 14]

$$
H(s, B)=\frac{B / B_{\mathrm{c}}}{\left[1-\left(T_{\perp} / T_{||}\right)\left(1-B / B_{\mathrm{c}}\right)\right]},
$$

as long as we choose the constant $B_{\mathrm{c}}<B_{\min }$, with $B_{\min }$ the minimum value of $B$ in the plasma. Furthermore, imposing that the perpendicular and parallel hot particle temperatures satisfy the condition $T_{\perp}=T_{\|}=T$, the form factor $H$ reduces to unity. The pressure thus becomes isotropic. This is the choice we have made for all of the work presented in this paper. We can analyse anisotropic pressure profiles, however it is expected that the ITER fast particle distribution function will remain relatively isotropic. Consequently, we can write

$$
p(s)=\mathcal{M}(s)\left[\Phi^{\prime}(s)\right]^{\Gamma} \frac{1+p_{\mathrm{h}}(s)}{\left\langle 1+p_{\mathrm{h}}(s)\right\rangle^{\Gamma}} .
$$

We select $p_{\mathrm{h}}$ to be a constant much smaller than unity, typically 0.001 , so that effectively $p(s) \simeq \mathcal{M}(s)\left[\Phi^{\prime}(s)\right]^{\Gamma}$. Note that $p=\mathcal{M}$ for $\Gamma \equiv 0$, however, we have made the choice $\Gamma=5 / 3$ and therefore prescribe the plasma mass profile and calculate the pressure profile accordingly. The major disadvantage is that the plasma mass is not an intuitively straightforward quantity to understand from an experimental perspective, at least compared with the pressure. On the other hand, the total system energy, as depicted in equation (1), 


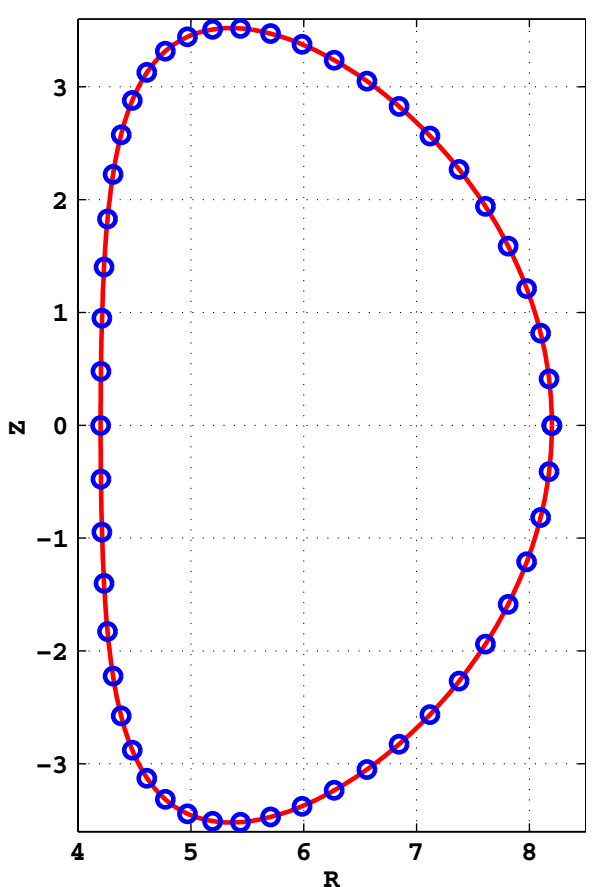

Figure 1. An up-down symmetric description of the ITER plasma boundary. The dimensions of $R$ and $Z$ are expressed in metres.

increases (decreases) for $\Gamma>1(\Gamma<1)$ as the plasma $\langle\beta\rangle$ becomes larger. The idea that the energy should decrease as we increment the $\langle\beta\rangle$ value is not very appealing. This is the reason we impose $\Gamma=5 / 3$.

\section{Inputs and profiles}

A Fourier decomposition of the plasma-vacuum interface must be provided to the ANIMEC code [9] for fixed boundary equilibrium computations. The description of an ITER boundary is presented in figure 1, which is then Fourier analysed to obtain the necessary input data. For simplicity, we have used an axisymmetric up-down symmetric description of the last closed flux surface. The Fourier coefficients of $R$ (the distance from the major axis) and $Z$ (the height from the midplane) at the plasma edge are obtained and presented in table 1 .

The ANIMEC code requires $\Gamma$, the edge toroidal magnetic flux, the plasma current and the parameter $B_{\mathrm{c}}$ and four profiles [9]. The first of these profiles is the plasma mass (the thermal pressure for $\Gamma \equiv 0)$. We specifically choose the following polynomial expansion:

$\mathcal{M}(s)=\mathcal{M}(0)\left(1+0.4102275 s^{2}-14.19875 s^{4}+29.62525 s^{6}-22.9512 s^{8}+6.1152 s^{10}\right)$.

We choose $\mathcal{M}(0)=4 \times 10^{4}$ to obtain equilibria with volume averaged $\langle\beta\rangle \simeq 2.9 \%$ when the toroidal magnetic flux enclosed within the plasma boundary $2 \pi \Phi(1)=105 \mathrm{~Wb}$. The toroidal magnetic field at the magnetic axis then is approximately $4.6 \mathrm{~T}$ and $\beta_{\mathrm{N}} \simeq 2.2$ when the toroidal current is $13 \mathrm{MA}$. The pressure profile obtained with the plasma mass expression given in equation (5) is shown in figure 2. It is flat in the central region of the plasma consistent with 
Table 1. The Fourier coefficients of $R$ and $Z$ that describe the last closed flux surface of an ITER configuration. The poloidal mode number is $m$ and the toroidal mode number is $n$. The dimensions of $R_{m n}$ and $Z_{m n}$ are expressed in metres.

\begin{tabular}{llll}
\hline$m$ & $n$ & \multicolumn{1}{c}{$R_{m n}(1)$} & \multicolumn{1}{l}{$Z_{m n}(1)$} \\
\hline 0 & 0 & 5.8722 & \multicolumn{1}{l}{0} \\
1 & 0 & 2.0291 & 3.5185 \\
2 & 0 & $3.1581 \times 10^{-1}$ & $-1.5611 \times 10^{-1}$ \\
3 & 0 & $-2.9946 \times 10^{-2}$ & $1.4543 \times 10^{-2}$ \\
4 & 0 & $1.3105 \times 10^{-2}$ & $1.2426 \times 10^{-2}$ \\
5 & 0 & $5.3984 \times 10^{-4}$ & $-2.9503 \times 10^{-3}$ \\
6 & 0 & $-1.2283 \times 10^{-3}$ & $-4.6531 \times 10^{-4}$ \\
7 & 0 & $3.7245 \times 10^{-4}$ & $7.6980 \times 10^{-4}$ \\
8 & 0 & $1.0836 \times 10^{-4}$ & $-1.4809 \times 10^{-4}$ \\
\hline
\end{tabular}

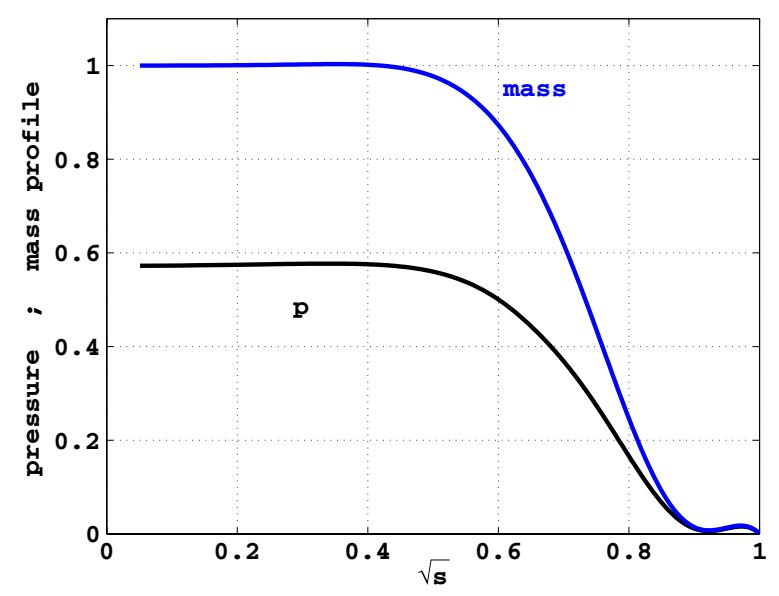

Figure 2. The pressure profile obtained using the mass profile prescribed by equation (5) and the mass profile as a function of $\sqrt{s}$. The pressure is given in units of pascals times $\mu_{0}=4 \pi \times 10^{-7} \mathrm{H} \mathrm{m}^{-1}$, the permeability of free space.

a large $q_{\min } \simeq 1$ radius and with observations in the TCV tokamak when sawteeth relaxations disappear $[15,16]$.

The second profile is the hot particle perpendicular to parallel temperature ratio $T_{\perp} / T_{\|}$ chosen as unity for isotropic pressure conditions, further guaranteed by selecting $B_{\mathrm{c}}=$ $0.026 T \ll B_{\min }$. The third profile of ANIMEC is $p_{\mathrm{h}}$ for which the constant 0.001 is given.

The fourth profile can be either the rotational transform $1 / q$ or the toroidal plasma current. It is easiest to prescribe inverse $q$. However, while the $q$-profile can be quite insensitive to details in the toroidal current profile, the converse does not hold. Imposing the $q$-profile can yield a toroidal current profile that reverses in the outer fraction of the plasma volume with a finite jump at the plasma-vacuum interface. Therefore, the toroidal current profile prescription tends to be preferable to guarantee physically realistic conditions. We have adapted and implemented in ANIMEC the model employed in the CHEASE code [17] to describe the toroidal current profile by three piecewise differentiable polynomials, rather than the standard polynomial expansion employed in VMEC [7]. From the magnetic axis to some surface labelled by $s_{1}$, the profile is quadratic, from $s_{1}$ to $s_{2}$ it is cubic, and linear from $s_{2}$ to the plasma edge $(s=1)$. There are seven coefficients to provide as input. These are the radial positions $s_{1}$ 


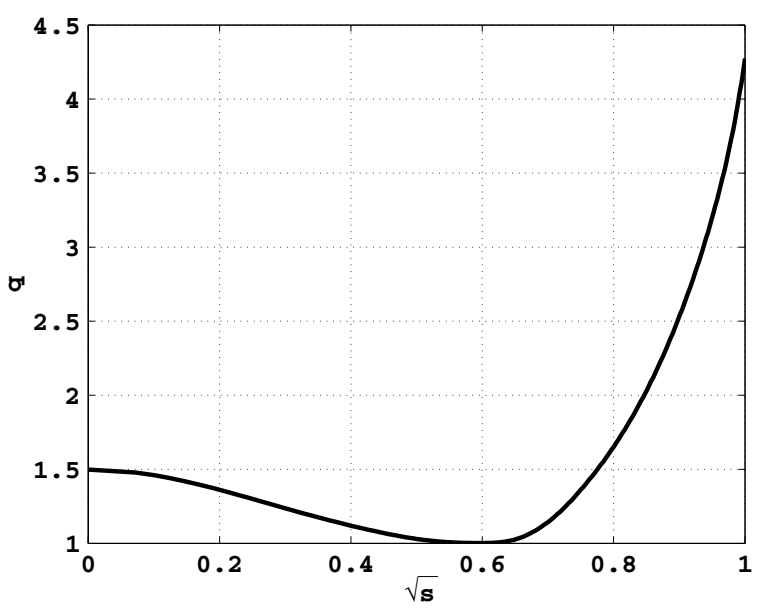

Figure 3. The inverse rotational transform profile $q$ as a function of $\sqrt{s}$ for the case with 13.3 MA toroidal current.
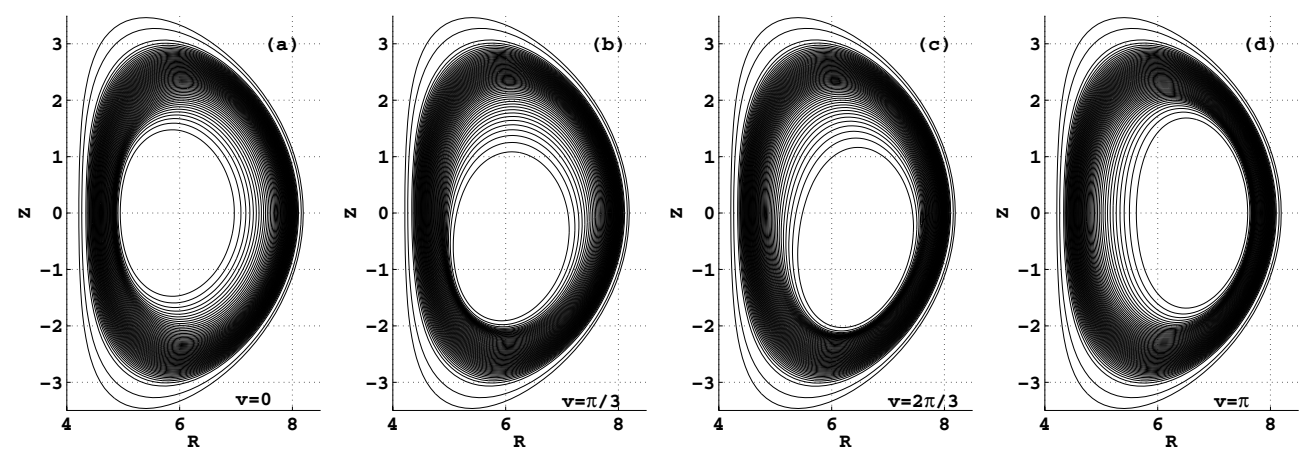

Figure 4. Contours of constant pressure at cross sections with toroidal angle $(a) v=0,(b)$ $v=\pi / 3,(c) v=2 \pi / 3$ and $(d) v=\pi$ for an ITER hybrid scenario equilibrium with 13.3 MA toroidal plasma current. $R$ and $Z$ are in metres.

and $s_{2}$, the values of $J^{\prime}(s)$ at the magnetic axis and at the plasma edge and the values of $J^{\prime \prime}(s)$ at the magnetic axis, at $s_{1}$ and at $s_{2}$. The toroidal plasma current enclosed within the surface $s$, which corresponds to $2 \pi J(s)$, is integrated analytically from the profile prescribed, it is normalized to its value at the plasma boundary and is multiplied by the total current which is a separate input parameter of the code. The current profile coefficients $J^{\prime}(0)=1, J^{\prime}(1)=0$, $J^{\prime \prime}(0)=5, J^{\prime \prime}\left(s_{1}\right)=-2$, and $J^{\prime \prime}\left(s_{2}\right)=-0.05$ have been fixed for all the calculations undertaken in this work. We have varied $s_{1}, s_{2}$ and adjusted the total plasma current so that the minimum value of $q\left(q_{\min }\right)$ is in the neighbourhood of unity. The corresponding $q$-profile for a case with toroidal current of 13.3 MA, $s_{1}=0.35$ and $s_{2}=0.55$ is shown in figure 3 . When $q_{\text {min }}$ is near unity at a sufficiently large plasma radius, we can obtain an equilibrium with a 3D helical core despite imposing a fixed axisymmetric plasma boundary [10]. An example with the ITER-shaped boundary shown in figure 1, with the pressure of figure 2 and $q$-profile of figure 3 , is given in figure 4 . These new equilibrium states can exist in a range of $q$-profiles having $q_{\text {min }} \simeq 1$. For the set of parameters used in this study, cases with 13.1 and 13.8 MA are presented in figure 5 that encompass a sequence of equilibria with $3 \mathrm{D}$ helical cores. 


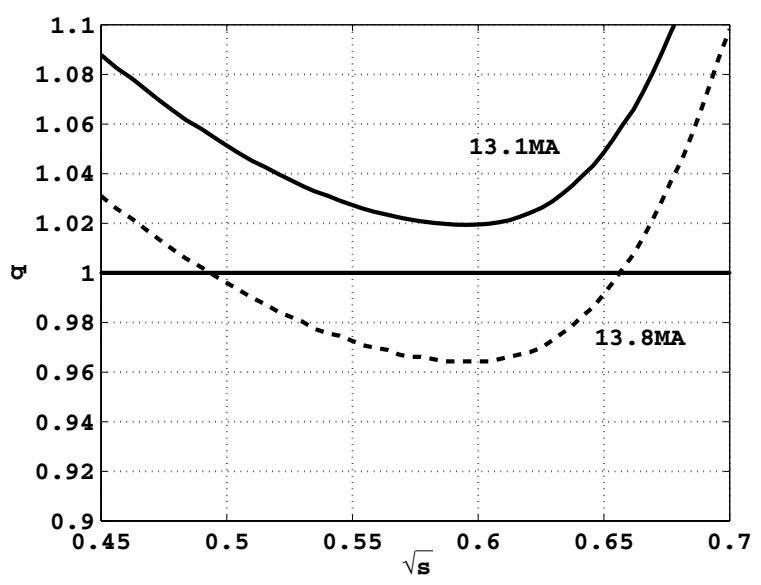

Figure 5. Zoom of the inverse rotational transform $q$-profiles as a function of $\sqrt{s}$ for the cases with toroidal current of 13.1 MA (solid curve) and 13.8 MA (dashed curve).

\section{Convergence properties of helical core equilibria}

\subsection{Convergence study with radial mesh}

We investigate the dependence of the extent of the helical deformation of the magnetic axis on the radial mesh size for a sequence where we vary the number of radial grid points from 81 to 209. The spectrum of Fourier components encompass $0 \leqslant m \leqslant 8$ and $-4 \leqslant n \leqslant 4$, where $m$ is the poloidal mode number and $n$ is the toroidal mode number. The interface locations of the current profile segments are given by $s_{1}=0.4$ and $s_{2}=0.6$. To obtain equilibria with $q_{\text {min }} \simeq 1$, we require a total plasma current of $15.1 \mathrm{MA}$.

We define the helical deformation of the magnetic axis as

$$
\delta_{\mathrm{H}}=\frac{\sqrt{R_{01}^{2}(s=0)+Z_{01}^{2}(s=0)}}{a},
$$

where $R_{01}\left(Z_{01}\right)$ is the ( $\left.m=0, n=1\right)$ Fourier component of $R(Z)$ at the magnetic axis and $a=2.65 \mathrm{~m}$ is the effective plasma minor radius. The extent of the helical axis distortion as a function of the inverse of the number of radial grid points $N_{r}^{2}$ is displayed in figure 6 . We conclude that the choice of $N_{r}>150$ radial mesh points constitutes an adequate number to obtain reliably converged helical ITER equilibrium solutions. We therefore employ $N_{r}=193$ for the calculations described in the next sections.

\subsection{Convergence study with poloidal and toroidal mode numbers}

The convergence of the helical core ITER equilibrium states with respect to the number of Fourier mode components is investigated in this section. For the sequence in which we vary the maximum poloidal mode number $m_{\max }$ from 8 to 11, we have fixed the maximum toroidal mode number $n_{\max }=4$, while that in which we alter $n_{\max }$ from 3 to 6 has fixed $m_{\max }=10$. The toroidal plasma current in these sequences is 13.2 MA. The helical distortion parameter $\delta_{\mathrm{H}}$ is displayed as functions of $m_{\max }$ and $n_{\max }$ in figure 7 from which we conclude that mode numbers $m_{\max }=8$ and $n_{\max }=4$ can reliably generate acceptably converged equilibrium structures. These limits are imposed for the computations presented in subsequent sections. 


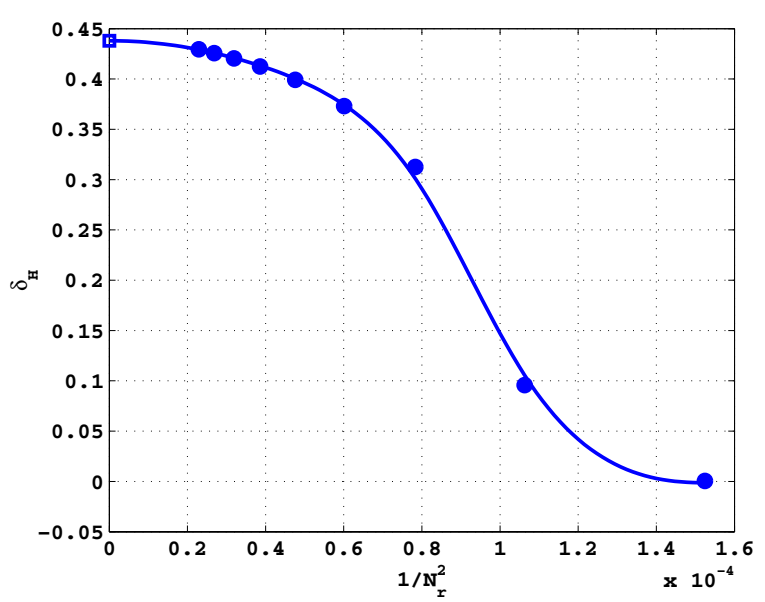

Figure 6. Convergence of the magnetic axis helical distortion parameter $\delta_{\mathrm{H}}$ as a function of the inverse number of radial grid points $N_{r}$ for an ITER equilibrium with 15.1 MA toroidal current. The value of $\delta_{\mathrm{H}}$ extrapolated to infinitesimal mesh size is $\sim 0.44$.
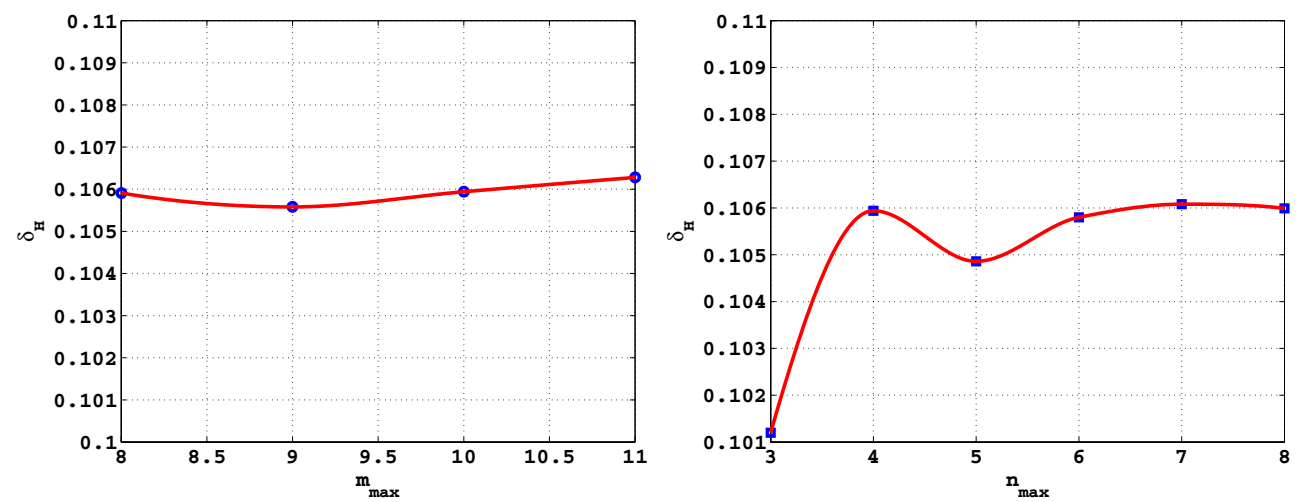

Figure 7. Convergence of the magnetic axis helical distortion parameter $\delta_{\mathrm{H}}$ with respect to the maximum poloidal mode number $m_{\max }$ (left) and with respect to the maximum toroidal mode number $n_{\max }$ (right) for an ITER equilibrium with 13.2 MA toroidal current.

Computer simulation programmes that apply a Fourier representation in the angular coordinates converge exponentially with the number of poloidal and/or toroidal grid points [18]. Typically, we need to choose the number of toroidal grid points $N_{\mathrm{t}}$ in the range $3 n_{\max }+2 \leqslant$ $N_{\mathrm{t}} \leqslant 4 n_{\max }+1$ and the number of poloidal grid points $N_{\mathrm{p}}$ in the range $4 m_{\max } \leqslant N_{\mathrm{p}} \leqslant 5 m_{\max }+1$ to guarantee correct solutions. The computations that follow with $m_{\max }=8$ and $n_{\max }=4$ have $N_{\mathrm{t}}=3 n_{\max }+2$ and $N_{\mathrm{p}}=5 m_{\max }$ which satisfy these criteria.

\section{Helical ITER equilibria}

\subsection{Toroidal plasma current scan}

We investigate a sequence of ITER equilibria where we choose $s_{1}=0.35$ and $s_{2}=0.55$ and vary the toroidal current between 13.1 and 13.8 MA. In this range, we obtain equilibria with 


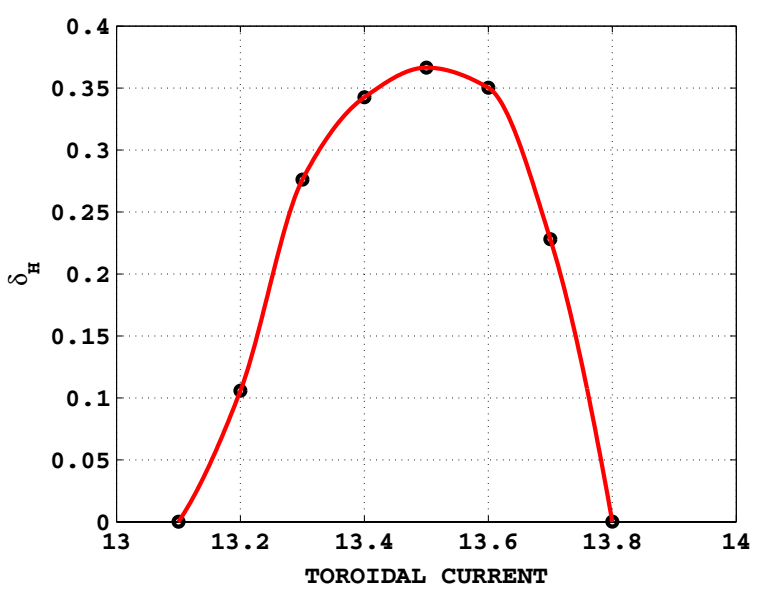

Figure 8. The magnetic axis helical distortion parameter $\delta_{\mathrm{H}}$ as a function of the toroidal plasma current in MA.

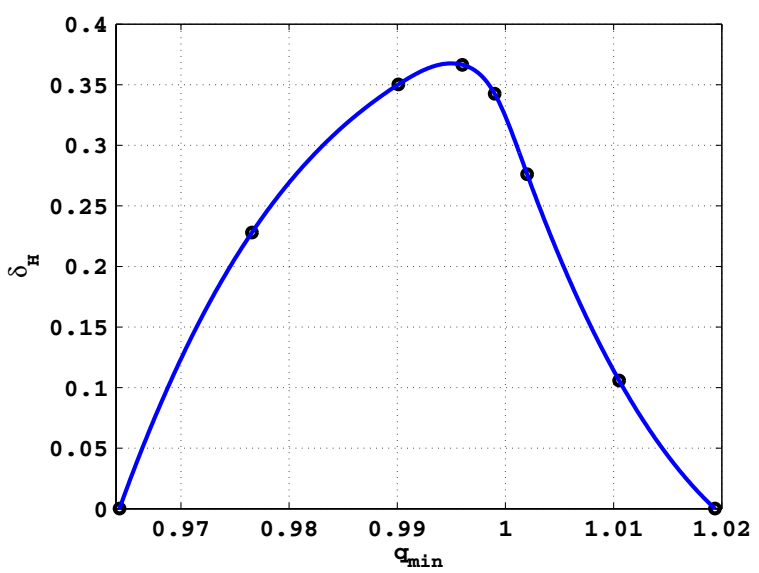

Figure 9. The magnetic axis helical distortion parameter $\delta_{\mathrm{H}}$ as a function of $q_{\mathrm{min}}$.

spontaneous 3D helical distortions of the core region of the plasma though the boundary, as it is fixed, remains axisymmetric.

The magnetic axis deformation $\delta_{\mathrm{H}}$ as a function of the toroidal plasma current is plotted in figure 8. As we increase the plasma current, the value of $q_{\mathrm{min}}$ decreases spanning the range shown in figure 5. We show the corresponding dependence of $\delta_{\mathrm{H}}$ with respect to $q_{\min }$ for the sequence we have studied in figure 9.

The contours of constant pressure at four toroidal cross sections spanning half of the torus were displayed in figure 4 for the case with 13.3 MA toroidal plasma current. We highlight the change in the internal helical structures with current by plotting the constant pressure contours at the toroidal angle $v=2 \pi / 3$ (one third of the way around the torus) for the sequence of equilibria described in figures 8 and 9 as we vary the current from 13.1 to 13.8 MA in figure 10 which confirm that the maximum helical deformation occurs at a current of 13.5 MA where $q_{\min } \simeq 0.996$. 

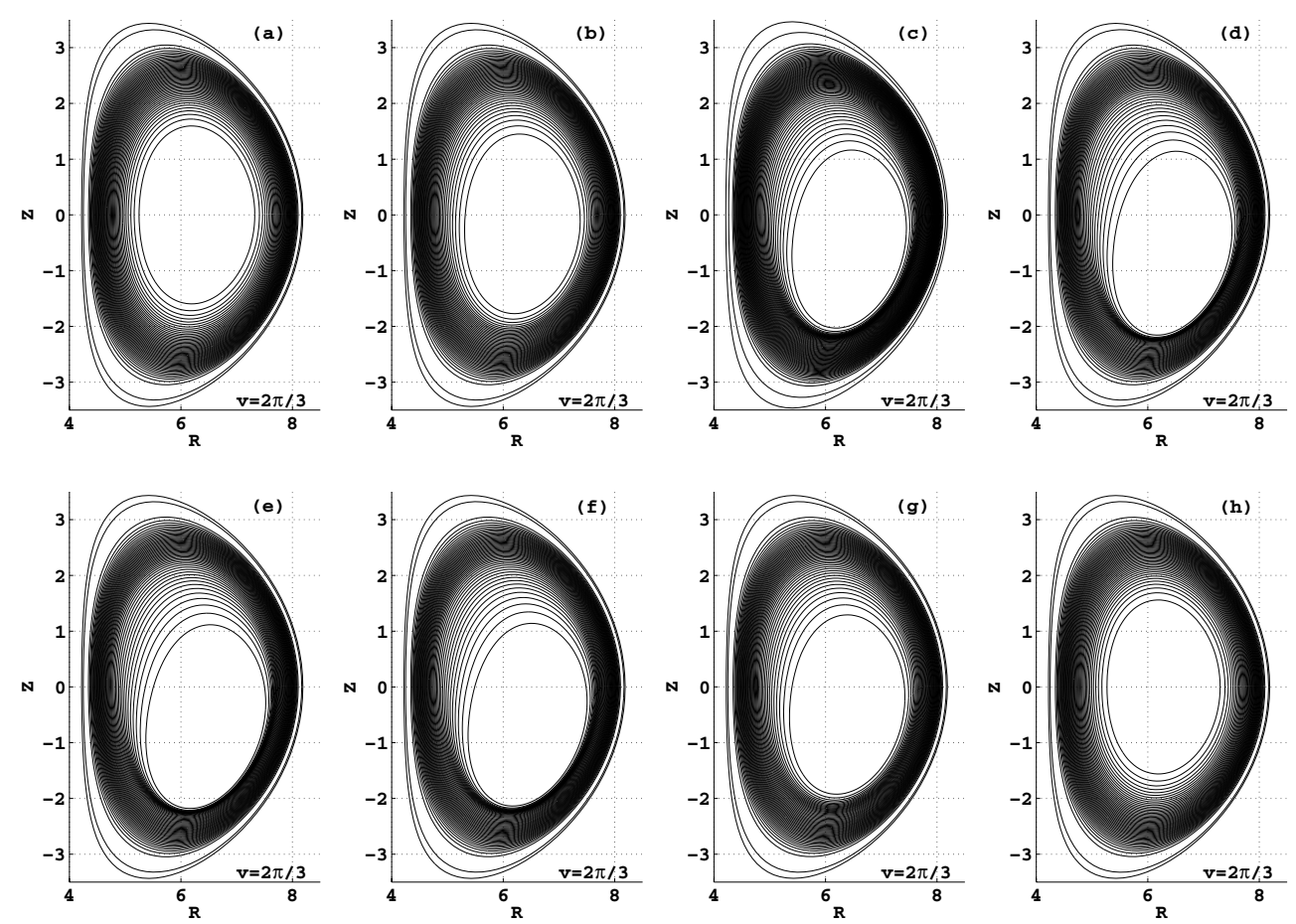

Figure 10. Contours of constant pressure at the cross sections with toroidal angle $v=2 \pi / 3$ with toroidal current (a) 13.1 MA, (b) 13.2 MA, (c) 13.3 MA, $(d)$ 13.4 MA, $(e)$ 13.5 MA, $(f)$ 13.6 MA, $(g) 13.7 \mathrm{MA}$ and $(h) 13.8 \mathrm{MA}$, where $R$ and $Z$ are expressed in metres.

\subsection{ITER hybrid scenario study: $\langle\beta\rangle$ scan}

The ITER hybrid scenario is specifically targeted to have a toroidal field of $5.3 \mathrm{~T}$ and toroidal plasma currents in the 12-14 MA range [12]. In order to achieve these targets, we have increased the toroidal magnetic flux to $121.6 \mathrm{~Wb}$ and sharpened the toroidal current profile by imposing $s_{1}=0.36, s_{2}=0.38, J^{\prime \prime}(0)=12$ and $J^{\prime \prime}\left(s_{1}\right)=-10$. The remaining coefficients for the current profile are the same as in the current scan we have described in the previous section. A sequence of equilibria is computed by changing $\mathcal{M}(0)$ from 2 to 50000 to obtain a finite $\langle\beta\rangle$ scan from 0 to $3.5 \%$ (corresponding to $\beta_{\mathrm{N}} \simeq 3.3$ ). The helical distortion of the magnetic axis is plotted in figure 11 showing that a helical core exists in the absence of plasma pressure and increases somewhat with $\langle\beta\rangle$. The toroidal plasma current in this sequence is fixed at $12 \mathrm{MA}$ and the number of radial grid points $N_{r}=289$.

\section{Conclusions and discussion}

The work presented in this paper represents a paradigm shift for tokamak magnetic confinement physics research because we have theoretically demonstrated that the equilibrium state in the ITER device operating in the hybrid scenario with $q_{\min } \simeq 1$ can develop an internal helical core structure with the essential characteristics of a saturated $m=1, n=1$ ideal internal kink mode. Scans in toroidal plasma current and $\langle\beta\rangle$ have been generated to determine the sensitivity and magnitude of the core helical deformations with respect to these parameters. The structures are computed in the 12-15 MA range. The toroidal current profile that is prescribed 


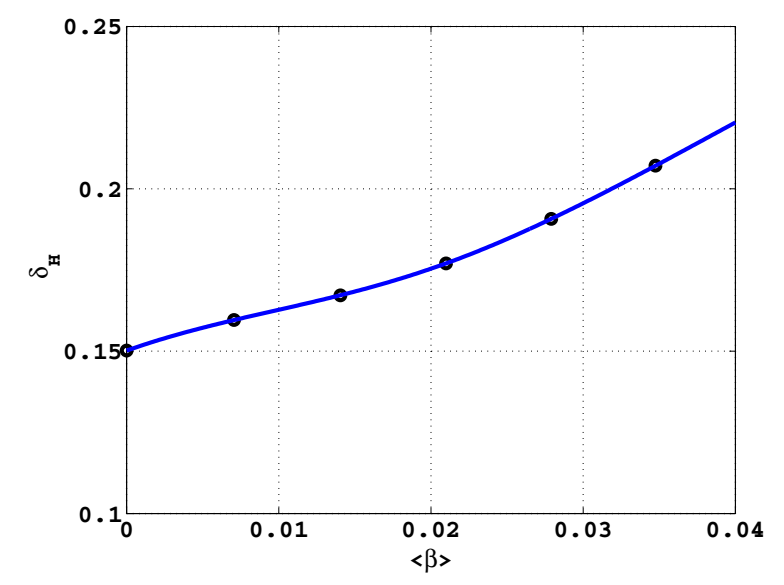

Figure 11. The magnetic axis helical distortion parameter $\delta_{\mathrm{H}}$ as a function of the volume averaged $\langle\beta\rangle$.

becomes more peaked towards the lower end of this range. However, we conjecture that the exact axisymmetric boundary we have imposed constitutes a sufficiently severe restriction that forces the helical core to develop above a relatively high current threshold. In future work, we shall concentrate on free boundary calculations to ascertain the robustness of the $3 \mathrm{D}$ core helical equilibrium states in a wider range of toroidal currents and profiles.

\section{Acknowledgments}

This research was partially sponsored by the Fonds National Suisse de la Recherche Scientifique and Euratom. The authors are very grateful to S P Hirshman for his invaluable contributions to the development of the 3D equilibrium solvers we use which has made this research possible. They also thank M Albergante for the ITER boundary.

(C) Euratom 2011.

\section{References}

[1] Weller A et al 1987 Phys. Rev. Lett. 592303

[2] Chapman I T et al 2010 Nucl. Fusion 50045007

[3] Puiatti M E et al 2004 Plasma Phys. Control. Fusion 51120431

[4] Avinash, Hastie R J, Taylor J B and Cowley S C 1987 Phys. Rev. Lett. 592647

[5] Lütjens H and Luciani J F 2008 J. Comput. Phys. 2276944

[6] Garabedian 2006 Proc. Natl Acad. Sci. USA 10319232

[7] Hirshman S P and Betancourt O 1991 J. Comput. Phys. 9699

[8] Terranova D et al 2010 Plasma Phys. Control. Fusion 52124023

[9] Cooper W A et al 2009 Comput. Phys. Commun. 1801524

[10] Cooper W A et al 2010 Phys. Rev. Lett. 105035003

[11] Gormezano C et al 2004 Plasma Phys. Control. Fusion 46 B435

[12] Gormezano C et al 2007 Nucl. Fusion 47 S285

[13] McClements K G et al 1996 Phys. Plasmas 32994

[14] Cooper W A et al 2006 Nucl. Fusion 46683

[15] Reimerdes H et al 2006 Plasma Phys. Control. Fusion 481621

[16] Camenen Y et al 2007 Nucl. Fusion 47586

[17] Lütjens H, Bondeson A and Sauter O 1996 Comput. Phys. Commun. 9547

[18] Schwenn U 1984 Comput. Phys. Commun. 31167 